\title{
Künstlerische Qualitätssicherung und Öffnung für neues Publikum. Berufung und Antritt einer Staatstheaterintendanz als kulturpolitische (Selbst-)Legitimation am Beispiel Hannover
}

\section{Sebastian Stauss}

\section{Zusammenfassung}

Die kulturpolitische und programmatische Ausrichtung des öffentlich getragenen deutschen Theaters wird selten deutlicher als zu Beginn von Intendanzen und Direktionen. So auch in Hannover: Für die Staatsoper nehmen seit dem Jahr 2000 ökonomische Abhängigkeiten durch aufgelaufene Defizite, Zielvorgaben und stagnierenden Kulturetat zu. Der Intendanzantritt 2019/2020 von Laura Berman an der Oper und Sonja Anders am Schauspiel stand im Zeichen einer stärkeren institutionellen Öffnung, wie diskursbezogen anhand von Presseartikeln und kulturpolitischen Statements genau nachvollziehbar ist.

\section{Schlüsselwörter}

Staatsoper $\bullet$ Hannover $\bullet$ Niedersachsen $\bullet$ Kulturpolitik $\bullet$ Intendanz

\section{Rahmen und Organisationsstrukturen}

Im Winter 2016 wurde die kulturpolitische Legitimation der Niedersächsischen Staatsoper massiv infrage gestellt - zumindest, wenn man Medienreichweite

\footnotetext{
S. Stauss $(\varangle)$ Deutschland

E-Mail: sebastian.stauss@1mu.de

Institut für Theaterwissenschaft, Ludwig-Maximilians-Universität München, München, 
und -echo von „Deutschlands populärste[m] Komiker“ (Arndt 2016), mit eigener Show im Privatfernsehen, als Maßstab zugrunde legt: Mario Barth war u. a. mit versteckter Kamera ins Feld gezogen, um am Beispiel einer (wohl wider Erwarten gut besuchten) Opernvorstellung Steuerverschwendung anzuprangern, angesichts der immergleichen Stücke im Repertoire, wie Mozarts Zauberflöte, vermeintlich geringen Interesses des ohnehin überalterten Publikums und zulasten von Einrichtungen im Bildungs- und Sozialwesen wie Kindergärten. Vonseiten des Verwaltungsdirektors des Staatstheaters, Jürgen Braasch, wurde als Stellungnahme hierzu nur Bedauern über eine vor der Reportage nicht erfolgte Kontaktaufnahme mit der Staatsoper kundgetan. Es hätte andernfalls gezeigt werden können, wie „effizient“ das Theater arbeite (Arndt 2016).

In jedem Fall bediente sich der brachiale Investigativ-Versuch eines Framings, das bezogen auf das deutsche Theater allgemein und besonders für die Staatsoper in Hannover als ,Fallbeispiel' 2016 schon angejahrt erschien. Von einseitiger, hierarchisch unflexibler Pflege eines elitären Repertoire- und Abonnementsystems kann in der niedersächsischen Landeshauptstadt spätestens seit 2001 allenfalls noch eingeschränkt die Rede sein, nachdem die Leitung des Opernhauses nach zwei Jahrzehnten unter dem Intendanten und Regisseur Hans-Peter Lehmann neu besetzt worden war. Die folgenden Spielzeiten unter Albrecht Puhlmann (bis 2006) waren von einer massiven Krise und Irritationen durch die künstlerische Programmatik nach außen geprägt, insbesondere durch Regieskandale wie durch Calixto Bieito oder das Infragestellen des traditionellen Opernbetriebes in Produktionen wie Europeras 1-5 von John Cage. Auch im Verhältnis zur Kulturpolitik kam es zu Spannungen: Nach eineinhalb Spielzeiten war 2003 rund ein Drittel der Abonnements gekündigt worden, was von Puhlmann u. a. darauf zurückgeführt wurde, dass man in Hannover zuvor bei wichtigen ästhetischen Entwicklungen „geschlafen“ hätte (TAZ 2003a). Im folgenden Sommer wurden angesichts eines angehäuften Staatstheater-Defizits von rund 3,5 Mio. EUR kulturpolitische Maßnahmen nötig (TAZ 2003b) - bei einem Staatstheater-übergreifenden Etat von damals rund 48 Mio. Die Konsequenz aus dem niedersächsischen Kulturministeriums: „Rund 3,5 Mio. EUR mussten als Sonderzuwendungen für das Staatstheater ,nachgeschossen“ werden." Zugleich wurde klargestellt, dass in den Spielzeiten danach die Vorgaben von Einsparungen (450.000 EUR jährlich bis 2006) sowie solideren Planens und Wirtschaftens gelten würden (TAZ 2003b). Die folgende Opernintendanz von Michael Klügl (2006-2019), weitgehend parallel zu jener von Lars-Ole Walburg am Schauspiel (2009-2019), unter denen der Etat auf über 75 Mio. anwuchs, stand auch im Zeichen wirtschaftlicher Konsolidierung.

Insofern, als in den frühen 2000er Jahren also wirtschaftliche Effizienz in Hannovers Staatsopernbetrieb notwendiger denn je wurde, trat auch die Rechtsform 
und Betriebsstruktur der Niedersächsischen Staatstheater GmbH umso stärker in den Vordergrund. Diese GmbH mit einem Aufsichtsrat als Steuerungsorgan ist seit 1970 an die Stelle der vormaligen Landestheater Hannover GmbH getreten und wird heute, wie es auf der Homepage des Niedersächsischen Ministeriums für Wissenschaft und Kultur heißt, ,aus historisch gewachsenen Gründen als 100-\%ige Gesellschaft des Landes betrieben“ (MWK Niedersachsen (o. J.)).

Die erwähnte wirtschaftliche Konsolidierung des Staatstheaters ging auch mit Zielvereinbarungen zwischen dem Staatstheater und dem Land Niedersachsen einher, die im Abstand von 3-4 Jahren neu formuliert werden. Im Unterschied zu anderen Bereichen (z. B. im Hochschulsektor) werden diese, der telefonischen Auskunft seitens des Ministeriums vom Februar 2020 zufolge, gerade hinsichtlich finanzieller Details nicht komplett veröffentlicht - einzelne Abschnitte wie beispielsweise zur Ausrichtung des Theater auf die Stadtgesellschaft oder auf Diversität sind aber bei sachgemäßer Anfrage einsehbar. Außer für die anderen Staatstheater-Betriebe in Niedersachsen (Braunschweig und Oldenburg) gelten im Bundesland auch Zielvereinbarungen für kommunale Theater (Schleiermacher 2013).

Im kulturpolitischen Trend liegt Hannover seit längerem aber nicht nur hinsichtlich dieses Aspektes der Governance, sondern auch in der programmatischen Ausrichtung auf ein jüngeres Publikum. Auch wenn die Zahl der Opernabonnements unter Klügl trotz gelegentlicher Ausschläge nach oben rückläufig blieb, stabilisierten sich die Zuschauerzahlen bei einer Auslastung zwischen $75 \%$ und $80 \%$, wobei insbesondere der erhöhte Verkauf von ermäßigten Karten für Schüler*innen, Studierende, Jugendliche und Kinder schon gegen Ende der Intendanz Puhlmann dokumentiert ist und die Gesellschaft der Freunde des Opernhauses, GFO-Hannover, ihr schon 1984 begonnenes Jugendförderprogramm „,Tatort Oper“ weiter vorantrieb (Barthold 2016; Timár 2017, S. 14 ff.).

Es bietet sich an, auch den Intendanzwechsel 2019 vor dem Hintergrund kulturpolitischer Legitimierungsmuster in und um die niedersächsische Landeshauptstadt zu betrachten. Die Berufung der neuen Doppelspitze in Hannover, Laura Berman als Opern- und Sonja Anders als Schauspielintendantin, ist als klares Statement der Gleichbehandlung zu sehen, der in den ersten Arbeitsschritten der Intendanz unter Gender- und Diversitätsaspekten weiterverfolgt worden ist. Die weitere Öffnung in gesellschaftlicher und ästhetischer Hinsicht ist mit Veränderungen in der Personalpolitik verknüpft.

Die folgende Betrachtung des Diskurses um die Berufung bezieht sich qualitativ vor allem auf Presseartikel (darunter mehrere Interviews und Statements), die in Relation zur niedersächsischen Kulturpolitik eingeordnet werden. Wie jedes Amt ist eine Intendanz angesichts zunehmend komplexer Verhältnisse, wie 
Albrecht Koschorke (2010, S. 52) anhand der Staatstheorie von Elman R. Service festgestellt hat, mit „dauerhafte[n] Sanktionsmittel[n]“, also legitimatorischen Verfahrensweisen ausgestattet. Zugleich ist und bleibt es aber ,als Elementarform politischer Institutionen, aus dem Imperativ der Vermittlung geboren" (Koschorke 2010, S. 52). Und so ist auch ein*e Intendant*in angesichts sich verlagernder Herausforderungen des Theaterbetriebs für die Erfüllung verschiedener Vermittlungsfunktionen verantwortlich: a) nach innen, zwischen künstlerischem und nicht-künstlerischem Bereich, spartenbezogen wie in Hannover mit der Besonderheit der musikalischen Leitungsebene im Musiktheater, b) nach außen hinsichtlich des Erfordernis eines klaren künstlerischen Profils und dramaturgischen Konzeptes, weitreichender Kommunikation (durch Öffentlichkeitsarbeit und Marketing) sowie zusätzlicher Angebote und Kooperationen (etwa im Bereich von Pädagogik und/oder kultureller Bildung sowie Sonderprojekten). Was die gesellschaftliche und (kultur)politische Legitimation betrifft, agiert ein*e Intendant*in außerdem nicht nur auf und zwischen diesen Ebenen, sondern zusätzlich c) auf der Ebene der primären und sekundären Stakeholder (Eschenbach und Horak 2003, S. 24), z. B. in Abstimmung mit kulturpolitisch Verantwortlichen, Publikum, Fördervereinen, Mäzen*innen und Sponsor*innen, wobei das Engagement von Letzteren im großstädtischen Musiktheaterbetrieb im Verhältnis zu anderen Sparten wiederum ein besonderes Gewicht hat.

Das folgende Beispiel von der Berufung bis zum Antritt der Staatstheaterintendanz in Hannover zwischen 2017 und 2019, mit besonderem Fokus auf der Opernintendantin Laura Berman, ist insofern bemerkenswert, als sowohl im Vorfeld als auch zu Beginn der Intendanz Modi der „Krisenvermeidung“ bei der Legitimierung durch Politik und designierte Theaterleitung erkennbar waren, sowohl die künstlerische Neuausrichtung als auch ihre Organisation im Theaterbetrieb betreffend.

\section{Programmatik und Personalpolitik}

Schon vor dem Amtsantritt der Intendantinnen Sonja Anders und Laura Berman in Hannover zum Spielzeitbeginn 2019/2020, gleich als die neue Doppelspitze designiert war, wurden von politischer Seite, gleichzeitig mit der Begründung der Berufung, Erwartungshaltungen formuliert. Nicht als „Geschlechtergerechtigkeit“, sondern als „,reine Bestenauslese““ (Meyer-Arlt 2017) bezeichnete die damalige niedersächsische Kulturministerin Gabriele Heinen-Kljajić von den Grünen die Entscheidung, unmittelbar nach der entscheidenden Aufsichtsratssitzung, präzisiert um die Begründung: „Beide verbinde[t] ein hoher künstlerischer Anspruch 
und die Überzeugung, dass die Öffnung von Oper und Schauspiel für neues Publikum von herausragender Bedeutung ist." (DPA 2017). Und an anderer Stelle wird Heinen-Kljajić zitiert: „Ich freue mich sehr, dass wir mit Laura Berman und Sonja Anders zwei außerordentlich innovative und hervorragend vernetzte Intendantinnen für Hannover gewinnen können“ (Hannover.de 2017). Wesentliche Punkte des Auftrags und der Erwartungen an beide sind damit klargestellt. Sie knüpfen zugleich bei der von der Ministerin drei Jahre zuvor umrissenen „konzeptbasierten Kulturpolitik“ an, die sie zum „,Kulturentwicklungskonzept Niedersachsen“" erläutert und für die sie die „Gewinnung neuer Publikumsschichten“ und die Formierung von „Netzwerke[n]“ zwischen Kulturschaffenden vor allem im Kontext von kultureller Bildung als zentrale Punkte hervorgehoben hat (Heinen-Kljajić 2014, S. 45). Der in der Landesverfassung (Artikel 72: „Besondere Belange und überkommene Einrichtungen der ehemaligen Länder“) begründeten, proportional besonders hohen Bezuschussung der Landeskultureinrichtungen (über $80 \%$ des Landeskulturetats) stehen seit den 1990er Jahren bei der Verteilung von Fördermitteln in Kooperation mit Landesverbänden und vereinigungen verstärkte Bestrebungen zur teilhabeorientierten Strukturförderung im soziokulturellen Bereich und in der kulturellen Bildung gegenüber. Für ,,[d]ie dialogorientierte Weiterentwicklung des ,Kulturentwicklungskonzeptes des Landes Niedersachsen“" (Heinen-Kljajić 2014, S. 40) hat somit der Austausch zwischen staatlichen und nicht-staatlichen Akteuren in städtischen und ländlichen Regionen an Bedeutung gewonnen.

Entsprechend rasch wurden nach der Berufung der neuen StaatstheaterIntendanz die Signale einer zeitgemäßen Theaterleitung ab 2019 ausgesendet. Dazu gehörte z. B. die Ausschreibung einer Stelle „Agent*in für Diversität“ mit der Förderung der Kulturstiftung des Bundes im Rahmen des Programms „,360Fonds für Kulturen der neuen Stadtgesellschaft“, die schließlich mit Leyla Ercan besetzt wurde (Hoffmann 2019). Das Vorab-Statement von Laura Berman hierzu lautete:

\footnotetext{
„Das Staatstheater Hannover sollte beispielhaft voranschreiten und einen offenen Prozess anstoßen, der hoffentlich auch über das Theater hinauswirkt. Ich bin gespannt, welche Entwicklung das Staatstheater in den nächsten Jahren nehmen wird. In jedem Fall profitieren wir alle: Hannover und die Region, unser Publikum und wir Theaterleute" (Staatstheater Hannover 2019).
}

Die kulturpolitische Legitimationsstrategie - gemeinsames Profitieren durch Weiterentwicklung des Theaters in Richtung der Übernahme gesellschaftlicher Aufgaben und Repräsentation von Diversität - ist anhand dieses Statements klar 
erkennbar. Von Bermans Kollegin im Schauspiel wird das Theater aber nicht nur in Anlehnung, sondern auch als Gegenpol zu gesellschaftlichen Strömungen begriffen, wie sie in Hinblick auf die Digitalisierung formuliert. Gerade hier erfahre Anders zufolge ,[d] as Schauspiel [...] einen enormen Bedeutungsgewinn. [...] In der Erfahrungswelt vieler junger Leute ist kaum noch Platz dafür, dass der Mensch [...] nicht dem normativen Schönheitsideal entspricht. [...] Theater ist der Ort, an dem der Mensch als Kreatur sichtbar wird. Das ist unschlagbar - und übertrumpft jede Virtualität.“ (Meyer-Arlt 2018). Alles andere als virtuell sind auch die an die Programmatik angepassten personellen Entscheidungen, wie die im Schauspiel auf 50 \% des Ensembles erhöhte Zahl von Schauspielerinnen und die Anhebung der Anzahl durch Frauen neu inszenierter Produktionen auf die Hälfte aller neuen Inszenierungen am Schauspiel.

In der Musiktheatersparte ist die Ensembleentwicklung etwas anders einzuordnen. Auf einen einzelnen Theaterbetrieb bezogen ist eine Reihe von Nicht-Verlängerungen beim Intendanzwechsel nichts Außergewöhnliches. Über den Einzelfall hinaus greift im Musiktheater seit Jahren jedoch ein bundesweiter Trend zur „Reduktion der Ensembles“ (Bertelsmann Stiftung 2019, S. 61 ff.) Unter Laura Bermans Vorgänger Michael Klügl war die Staatsoper Hannover mit 34 Stellen für fest verpflichtete Sänger*innen als ,immer noch eines der größten Ensembletheater hier in Deutschland“" angeführt worden (Strünkelnberg 2014). Unter Berman ist diese Zahl auf 26 gesunken, was sie mit dem Recht des Publikums auf Veränderung begründet hat: „Die Menschen in einer Stadt sollten die Gelegenheit erhalten, neue Sänger und neue künstlerische Handschriften kennenzulernen." (Arndt 2018). Ein zusätzliches Argument hierfür fand Berman auf der Ebene der Flexibilisierung:

\footnotetext{
„Natürlich braucht ein Haus von der Größe Hannovers ein Ensemble. Aber es gibt auch den Wunsch, Partien wirklich stilistisch passgenau zu besetzen. Dazu muss man dann auch mal Spezialisten engagieren. Es ist auch gar nicht so leicht, Sänger fest an ein Haus zu binden. Da kommt man manchmal mit regelmäßigen Gästen oder Teil-Engagements weiter" (Brandenburg 2018, S. 21).
}

Personelle Änderungen bzw. größere Einschnitte werden also mit ästhetischen Gründen legitimiert. Institutionentheoretisch gesehen wird hier jedoch ein Vorgang auf die Ebene der Legitimation gerückt, der nach Lynne G. Zucker (1988) an einer anderen Stelle institutioneller Muster in sozialen Zusammenhängen zu sehen ist. Institutionalisierung wird nach Zucker (ebd.) u. a. durch contagion, die soziale „Ansteckung“ von Legitimität, befördert, also durch bestimmte Werte oder (moralische oder sogar ideologische) Begründungszusammenhänge. Wenn, 
wie im Fall von Berman, der personelle Wandel am Theater über künstlerische Ansprüche bzw. solche des Publikums begründet wird, liegt diese Argumentation nach Zuckers Systematisierung von maintenance (der Aufrechterhaltung institutioneller Strukturen) vielmehr im Bereich jener Faktoren, die dem institutionellen Zusammenhalt entgegenwirken und ihn schwächen können - nämlich von Einzelinteressen und machtbündelnden Koalitionen. Im künstlerischen Bereich ist dies eher durch Kollektive wie Chor und Orchester und ihre Interessenvertretungen gegeben; aber auch Ensemblekonstellationen können eine Macht erlangen und geltend machen - wenn z. B. Solist*innen unter Vertrag stehen, die einen bestimmten Repertoirebereich abdecken (wollen), und andere Bereiche aus Budgetgründen nicht entsprechend realisierbar sind. In einem solchen Fall bilden sich über Ensemblestrukturen auch Spielplan- und Repertoirelinien heraus, wozu die Beobachtung des Augsburger Operndirektors Daniel Herzog passt, „dass viele Häuser weg vom Ensemble gehen, um sich künstlerisch freier entfalten zu können." (Bertelsmann Stiftung, S. 63). Gerade auf Freiheit und Flexibilisierung jenseits von (Fach-)Kategorien zielen Argumente von Berman, etwa wenn sie die vielerorts beschworene ,flache Hierarchie mit hoher Transparenz [...], die den Mitarbeitern Eigenverantwortung und Teilnahme am Prozess ermöglicht und ihnen das Gefühl gibt, viel Raum zu haben“, als Ziel ausgibt (Moghimi 2018); und wenn sie an anderer Stelle betont: „Wenn Künstler mit ihren Ideen zu mir kommen, merke ich, dass sie nicht mehr in den althergebrachten Kategorien denken.“ (Otten 2019, S. 120).

\section{$3 \quad$ Anpassung an die politische und mediale Gesamtstrategie}

Im eingangs bereits zitierten Text zum Staatstheater auf der Homepage des Niedersächsischen Ministeriums für Wissenschaft und Kultur, ist vermerkt:

„In den vergangenen drei Jahren haben die drei niedersächsischen Häuser ihren Kurs der konsequenten Öffnung der Häuser für neue Publikumsschichten fortgesetzt. [...] Im Mittelpunkt der Arbeit der Staatstheater, aber auch der Landes- und Stadttheater stehen die Entwicklung und Präsentation von qualitativ hochwertigen Theaterproduktionen. Dieses ,Kerngeschäft' wird in den großen und mittleren Städten seit einigen Jahren von vielfältigen, vor allem theaterpädagogischen Vermittlungsangeboten ergänzt. Einige Häuser bieten Interessierten darüber hinaus die Möglichkeit, sich in Chören oder Theaterclubs selbst künstlerisch zu betätigen“ (MWK Niedersachsen o. J.). 
Diesem Anspruch ist die Intendanz letztlich verpflichtet und muss hinsichtlich der Gewichtung zwischen Kerngeschäft und zusätzlichen Vermittlungsangeboten überregionaler und regionaler Bedeutung, den sich bietenden, von Berman im Sinne flacher Hierarchien reflektierten (Spiel)Raum zu nutzen verstehen. Wie sie es selbst formuliert, muss Berman ,[a]ls Theaterleiterin [...] Theater für Menschen machen, die vor Ort sind - aber nicht nur für diejenigen, die schon im Saal sitzen, sondern für diejenigen, die diesen Weg noch vor sich haben. Welche Themen, welche Narrative sind relevant für die Menschen, die in der Stadt leben, in der ich arbeite?" (Otten 2019, S. 119).

Bedingt durch den Koalitionswechsel von der rot-grünen zur rot-schwarzen Landesregierung in Niedersachsen im Herbst 2017, übernahm Sabine Johannsen, Staatssekretärin im nunmehr CDU-geführten Wissenschafts- und Kulturministerium, den Vorsitz im Aufsichtsrat der Staatstheater GmbH. Hinsichtlich des kulturpolitischen Auftrags der Theater bedeutet dies aber, was die in den zitierten Verlautbarungen betonte Kontinuität ,der konsequenten Öffnung der Häuser für neue Publikumsschichten" betrifft, keinen Bruch, allenfalls eine (Rück)Verlagerung hinsichtlich der Legitimationsebenen. Den Bezugsrahmen dafür stellen die als Kerngeschäft festgelegten „hochwertigen Theaterproduktionen“ und die „regionale und überregionale Bedeutung“, die als Postulate unmissverständlich klar machen, dass sich das Staatstheater nicht zuletzt in einem qualitätsbezogenen Wettbewerb befindet. Anlässlich des gemeinsamen Festumzugs von Staatsoper und Schauspiel in Hannover zur Spielzeiteröffnung Ende August 2019 formulierte der (CDU-)Landesminister für Wissenschaftler und Kultur Björn Thümler über die beiden Intendantinnen, quasi zwischen Statement und Erwartungshaltung: „Sie verleihen dem Spielort Hannover neuen Glanz.“ (Arndt 2019). Und Bermans Aussage am Rand derselben Veranstaltung mutet wie eine Modifizierung der vorangegangenen Legitimationsstrategie (aus ihrer Berufungsphase) an: „,Wir wollen etwas offener und informeller werden“, sagte sie - ,und trotzdem große Kunst bieten.““ (Arndt 2019). Letzteres ist keine geringe Herausforderung, da der Landesetat für Kultur im Vorfeld nicht erhöht, sondern trotz steigender Fixkosten auf dem gleichen Niveau gehalten wurde - ein Vorgang, der noch im Oktober 2018 eine Demonstration von 500 Kulturschaffenden vor dem niedersächsischen Landtag auf den Plan gerufen hatte. Neben der Initiative \#rettedeintheater war hier auch die Orchester Konferenz Niedersachsen aktiv (eine Vereinigung in niedersächsischen Kulturorchestern tätiger Musiker*innen). Vom landesweiten jährlichen Mehrbedarf von rund neun Millionen Euro für die letzten zehn Jahre primär betroffen sind die kommunalen Akteure, z. T. in existenzbedrohendem Maß z. B. Theater und Symphoniker in 
Lüneburg oder das Göttinger Symphonie-Orchester (DOV 2018). Aber das verknüpfte Problem des Ausgleichs von Tariferhöhungen bildet sich auch in der Landeshauptstadt ab, wie die Lageberichte der Niedersächsischen Staatstheater zum Jahresabschluss dokumentieren. Dort wurde für das Jahr 2017 festgehalten, dass ,auf Basis einer im Januar 2016 geschlossenen Zielvereinbarung die Mehrkosten aus den Tarifabschlüssen bis 2019“ vom Land Niedersachsen übernommen würden (Bundesanzeiger 2019) ${ }^{1}$. Ebenfalls 2017 wurden im Vergleich zum Vorjahr (Jahrbuch Opernwelt 2016, S. 148 und 2017, S. 148) sieben Orchesterstellen weniger verzeichnet (105 statt 112), die seither auch nicht nachbesetzt worden sind. Ein neuer Generalmusikdirektor wurde im Februar 2020 nach einjähriger Vakanz mit Stephan Zilias benannt (Hannover.de 2020).

Auch ohne Besetzung dieser Position ist Bermans Intendanz effizient gestartet: Gegenüber 16 Produktionen in der Saison 2018/2019 waren 15 für 2019/2020 angesetzt, davon mit 7 Premieren eine mehr im Großen Haus, ein erweitertes Nebenspielstätten-Angebot (vor allem für Kinder) und eine Premiere als Gemeinschaftsproduktion mit dem Schauspiel. Bei den Neuinszenierungen ist überdies eine Internationalisierung der Stückauswahl erkennbar, ohne dass sich die Gewichtung der Gattungen (Barockoper, Große Oper des 19. Jahrhunderts, Operette, Oper des frühen 20. Jahrhunderts und der Nachkriegszeit) wesentlich verschiebt.

Hinsichtlich der Etat-Problematik hat sich Laura Berman früh auf Diplomatie festgelegt. Die Frage in einem Interview, ob sie mit ihrem Etat auskomme, beantwortete sie wenige Wochen vor Beginn der ersten Spielzeit mit: „Ich denke ja. Wir planen schon bis in die dritte Spielzeit - mit einigen ganz großen Sachen. Ich verstehe allerdings, wenn die Politiker sagen, dass das Geld gebraucht wird für Krankenhäuser, Hochschulen. Aber ich finde, man darf das nicht gegen die Oper ausspielen.“ (Queren 2019) Statt der Forderung zusätzlicher Mittel setzt Berman hier quasi auf einen erinnernden Hinweis zum historisch begründeten deutschsprachigen Verständnis von Kultur als Teil der öffentlichen Daseinsvorsorge, über das auch eine Abgrenzung öffentlich getragener Kultureinrichtungen von der Kulturindustrie bei gleichzeitiger Wahrung von Innovationspotentialen und Stärkung von kultureller Bildung zum Ziel erklärt wurde, und zwar auf allen föderalistischen Ebenen (vgl. Deutscher Kulturrat 2004).

Ein biographisches Detail, auf das von und über Laura Berman in diesem Zusammenhang bereits wiederholt hingewiesen worden ist, ist ihre Herkunft ,aus

\footnotetext{
${ }^{1}$ Vorbereitet wird dieser Passus u. a. mit dem Hinweis: „Die deutschen Theater und Orchester haben in den letzten 20 Jahren erhebliche Konsolidierungsanstrengungen unternommen, was auch mit einem Personalabbau einherging."
} 
einer Familie mit lauter Pädagogen“ (Brandenburg 2018, S. 21). Die Pflege von partizipativen Musiktheaterprojekten, vor allem mit jungen Teilnehmer*innen, zählte ebenfalls, wie bereits das spartenübergreifende Arbeiten, schon in ihrer vorangegangenen Operndirektionszeit in Basel zu Bermans Referenzen. Auch hier besteht Kontinuität in der Vermittlung bezogen auf ihren Vorgänger Klügl, in dessen Intendanz 2010 die Junge Oper Hannover formiert wurde und schon vorher interkulturelle und partizipative Projekte mit Laien (wie 2008 Culture-Clash:Die Entführung) zu einer Spielplansäule wurden (Tamara Schmidt 2019, S. 154 ff.).

\section{$4 \quad$ Fazit}

Quasi vom Moment der Designation für das Amt der Intendantin an erfolgte auch Laura Bermans öffentliche Legitimierung in Hannover, und zwar als Vermittlerin auf allen drei eingangs eingeführten Ebenen (nach innen und nach außen sowie zwischen Stakeholdern). Obwohl dieses Bündeln von Vermittlungsfunktionen verglichen mit der Regietätigkeit anderer Amtsinhaber*innen weniger leitend als eben verbindend wirkt, erscheinen die (wiewohl punktuell anders gelagerten) Entscheidungsspielräume und Verantwortungsbereiche keinesfalls geringer. Erkennbar sind Prinzipien der maintenance nach Lynne G. Zucker, also der Erhaltung institutioneller Strukturen eines sozialen Systems, bei gleichzeitigen Gefährdungen der Institution durch konkurrierende Interessen, die eben der Moderation und Mediation bedürfen. Berufung und Antritt der Intendanz in Hannover lassen sich basierend auf dem maintenance-Konzept klar als Beispiel für eine Modifikation institutioneller Regeln und Rollenfestlegungen hinsichtlich Verantwortlichkeiten, Hierarchien und Vermittlung als triadischer Interaktion werten (Offe 1996, S. 203 f.), gegenüber der die nicht-modifizierte Aufrechterhaltung eine ungebremste Erosion der Legitimation erwarten ließe (Zucker, S. 26). Für eine weiterführende Analyse unter Zugrundelegung dieses institutionentheoretischen Ansatzes bietet sich die Betrachtung von der Intendanz untergeordneten Bereichen (auf der betrieblichen Mikroebene) ebenso an wie der Vergleich mit den vorangegangenen Intendanzen und graduellen oder versäumten Modifikationen.

Diskursstrategisch lässt sich zu Beginn dieser Staatsopern-Intendanz nicht nur eine ,solide“ Bilanz der ersten 100 Tage (Arndt und Meyer-Arlt 2019) und die Vertragsverlängerung des Verwaltungsdirektors Jürgen Braasch im Hintergrund festhalten (HAZ 2019), sondern auch eine solide Einstufung der Vorbereitungsphase. Im Unterschied zur Krise zu Beginn des Jahrtausends wurde bei der Vermittlung nach außen auf konzeptionelle Kontinuität in der Spielplangestaltung 
gesetzt und das Potenzial der Publikumserweiterung betont. Das spartenübergreifende und interdisziplinäre Arbeiten mit dem Schauspiel wird als Ziel der Vernetzung nach innen und zur Stadtgesellschaft hin projektweise initiiert, auf Potenzial zur Weiterentwicklung hin überprüft und Personalabbau - ebenso wie die technischen Erneuerungen sowie im Servicebereich (Queren 2019) - als Flexibilisierungsmaßnahmen umgesetzt. Nicht zuletzt wurden Signale an die politischen Entscheidungsträger*innen und Stakeholder gesendet, dass bei allem Effizienzstreben die historische Legitimation und Aushandlung (ausgehend von der Landesverfassung) die entscheidende Grundlage für den Staatstheaterbetrieb darstellt. Gerade für den bundesweiten Vergleich ist bemerkenswert, dass dabei von allen zitierten Akteuren Klarheit über die kulturpolitischen Diskursebenen besteht, auf denen die für ein Staatstheater erforderlichen Modifikationen, aber auch Kontinuitäten ausgehandelt werden. Konflikt- oder Krisenpotentiale, die in anderen (eingangs erwähnten) Fällen rasch nach dem Intendanzantritt zum Tragen kamen, wirken gezielt entschärft. Das Intendantinnenduo in Hannover kann insofern in seiner vermittelnden Funktion gemäß den (vom Zeitpunkt der Ernennung an keineswegs gleichbleibenden) kulturpolitischen Erwartungshaltungen auch nach einem auf Max Weber zurückgehenden und in der heutigen Kultursoziologie aufgegriffenen Grundprinzip eingeordnet werden: und zwar jenem der ideenbasierten „Weichenstellung“ (Weber 1989, S. 101; Griswold 2013, S. 38): Je nachdem, an welchem Punkt aus kulturpolitischer Perspektive eine Anpassung an die Dynamik materieller Interessen (ökonomischer Effizienz) und ideeller Interessen (gesellschaftlicher Öffnung) vonnöten ist, nehmen die beiden Theaterleiterinnen ihren intellektuellen, kulturellen und sozialen Kompetenzen gemäß entsprechende Kurskorrekturen vor. Zugleich erfordert die erhöhte Dynamik der Interessen immer neue und flexible Ideen der Gestaltung und des Agierens nach diesem weichenstellenden Prinzip.

\section{Literatur}

Arndt, Stefan. 2016. Alles nur junge Leute hier. Mario Barth war in Hannover in der Oper - mit versteckter Kamera, Hannoversche Allgemeine Zeitung 1. Dezember 2016.

Arndt, Stefan. 2018. Es ist wie in der Achterbahn (Interview mit Laura Berman). Hannoversche Allgemeine Zeitung 17. Juli 2018.

Arndt, Stefan. 2019. Ein starkes Fest. 8000 Besucher bei Spielzeitstart von Oper und Schauspiel. Hannoversche Allgemeine Zeitung 02. September 2019.

Arndt, Stefan. und R. Meyer-Arlt. 2019. Auftakt nach Maß. Hannoversche Allgemeine Zeitung 26. November 2019. 
Barthold, H.-M. 2016. Staatstheater Hannover - Arbeiten in einem kulturellen Hochleistungszentrum. Berufsreport https://www.berufsreport.com/staatstheater-hannover-arbeiten-ineinem-kulturellen-hochleistungszentrum/. Zugegriffen: 10. Febr. 2020.

Stiftung, Bertelsmann, Hrsg. 2019. Opernsänger mit Zukunft! Gütersloh: Verlag Bertelsmann Stiftung.

Brandenburg, D. 2018. I want to be in a soundworld for a while (Interview mit Laura Berman). Die deutsche Bühne 5: 16-21.

Bundesanzeiger. 2019. Niedersächsische Staatstheater Hannover Gesellschaft mit beschränkter Haftung Hannover: Jahresabschluss zum Geschäftsjahr vom 01.01.2017 bis zum 31.12.2017. Veröffentlichungs-Datum 19. Febr. 2019

Deutscher Kulturrat. 2004. Kultur als Daseinsvorsorge! Deutscher Kulturrat. https://www.kul turrat.de/positionen/kultur-als-daseinsvorsorge/. Zugegriffen: 10. Febr. 2020.

DOV (Deutsche Orchestervereinigung). 2018. Kommunale Orchester und Theater in Niedersachsen pochen auf Einhaltung des Koalitionsvertrags. Pressemitteilung 27. Sept. 2018.

DPA. 2017. Gleich zwei Intendantinnen am Staatstheater Hannover. Nordwest-Zeitung 12. Mai 2017.

Eschenbach, R., und C. Horak. 2003. Führung der Nonprofit-Organisation: bewährte Instrumente im praktischen Einsatz, 2. Aufl. Stuttgart: Schäffer-Poeschel.

Griswold, Wendy. 2013. Cultures and Societies in a Changing World, 4. Aufl. Los Angeles: Sage.

Hannover.de. 2017. Intendantenwechsel an Staatsoper und Schauspiel Hannover. https:// www.hannover.de/Service/Presse-Medien/Hannover.de/Aktuelles/Kultur-Freizeit-2017/ Intendantenwechsel-an-Staats\%C2\%ADoper-und-Schauspiel-Hannover. Zugegriffen: 10. Febr. 2020.

Hannover.de. 2020. Staatsoper Hannover: Stephan Zilias neuer Generalmusikdirektor. https:// www.hannover.de/Service/Presse-Medien/Hannover.de/Aktuelles/Kultur-Freizeit-2020/ Staatsoper-Hannover-Stephan-Zilias-neuer-General\%C2\%ADmusik\%C2\%ADdirektor. Zugegriffen: 9. März 2020.

HAZ. 2019. Staatstheater verlängert mit Braasch. Hannoversche Allgemeine Zeitung 06. Dezember 2019.

Heinen-Kljajić, Gabriele. 2014. Strukturbezogene Kulturförderung des Landes Niedersachsen. In Jahrbuch für Kulturpolitik, Bd. 14, Hrsg. Kulturpolitische Gesellschaft, 37-46. Essen: Klartext.

Hoffmann, Nina. 2019. Diversität muss normal werden. Leyla Ercan setzt sich als Agentin für Diversität am Staatstheater Hannover für die interkulturelle Öffnung des Hauses ein (Interview). TAZ, 23. Dezember 2019.

Koschorke, A. 2010. Institutionentheorie. In Die Figur des Dritten, Hrsg. E. Esslinger, T. Schlechtriemen, D. Schweitzer, und A. Zons, 49-64. Berlin: Suhrkamp.

Meyer-Arlt, Ronald. 2017. Damenwahl. Hannoversche Allgemeine Zeitung 12. Mai 2017.

Meyer-Arlt, Ronald. 2018. Ein Theater ist doch kein Museum (Interview mit Sonja Anders). Hannoversche Allgemeine Zeitung 30. April 2018.

Moghimi, J. 2018. Es gibt keine Diven (Interview mit Laura Berman). https://www.kul turmanagement.net/Themen/Interview-ueber-Fuehrung-im-Opernbereich-Es-gibt-keineDiven,2310. Zugegriffen: 10. Febr. 2020. 
MWK Niedersachsen (o. J.). https://www.mwk.niedersachsen.de/startseite/kultur/kulturspa rten/theater/staatstheater/niedersaechsische-staatstheater-19028.html. Zugegriffen: 10. Febr. 2020.

Offe, Claus. 1996. Designing institutions in East European transitions. In The theory of institutional design, Hrsg. Robert E. Goodin, 199-226. Cambridge: Cambridge University Press.

Opernwelt (2016 und 2017). Jahrbuch. Berlin: Der Theaterverlag.

Otten, Jonas. 2019. Wir sind doch keine Konzeptspuckapparate (Werkstattgespräch mit Laura Berman, Nicola Hümpel und Ann-Christine Mecke). In: Opernwelt Jahrbuch, 114-121. Berlin: Der Theaterverlag.

Queren, Henning. 2019. Ich spüre die positive Energie (Interview mit Laura Berman). Neue Presse Hannover, 29. August 2019, 20.

Schleiermacher, B. 2013. Große regionale Unterschiede im „Musikland Niedersachsen“. In: Recherchen in einem Theaterland. Die sechzehn Theater- und Orchestersystem der Bundesrepublik Deutschland im Vergleich, Hrsg. T. Schmidt, 193-207. ConBrio Verlagsgesellschaft: Regensburg.

Schmidt, Tamara. 2019. Interkulturalität im zeitgenössischen Theater für junges Publikum. In: Praxishandbuch Musiktheater für junges Publikum, Hrsg. C. Plank-Baldauf, 149-162. Berlin: Metzler \& Bärenreiter.

Staatstheater Hannover. 2019. Neue Intendanz setzt verstärkt auf Öffnung und Diversität. Staatstheater Hannover schreibt neue Stelle Agent*in für Diversität aus. Pressemitteilung 13. Februar 2019.

Strünkelnberg, Thomas. 2014. Eigenes Ensemble oder Starzirkus? Südwest Presse 13. August 2014.

TAZ. 2003a. Trillerpfeifen in der Oper. TAZ 07. April 2003.

TAZ. 2003b. Der Vorhang zu? TAZ Bremen 02. Juli 2003.

Timár, Z. 2017. Das Jugendförderprogramm der Opernfreunde Hannover. Eine qualitative Befragung ehemaliger Programmteilnehmer zu ihrem Verhältnis zur Oper heute. (Unveröffentlichte Masterarbeit, Hochschule für Musik, Theater und Medien Hannover).

Weber, Max. (1989). Die Wirtschaftsethik der Weltreligionen: Vergleichende religionssoziologische Versuche. Einleitung. In: $M W G, I / 19$, Hrsg. H. Schmidt-Glintzer, 83-127. Mohr! Siebeck: Tübingen.

Zucker, Lynne G. 1988. Where do institutional patterns come from? Organizations as actors in social systems. In Institutional patterns and organizations: culture and environment, Hrsg. L.G. Zucker, 23-49. Cambridge, Mass: Ballinger. 
Open Access Dieses Kapitel wird unter der Creative Commons Namensnennung 4.0 International Lizenz (http://creativecommons.org/licenses/by/4.0/deed.de) veröffentlicht, welche die Nutzung, Vervielfältigung, Bearbeitung, Verbreitung und Wiedergabe in jeglichem Medium und Format erlaubt, sofern Sie den/die ursprünglichen Autor(en) und die Quelle ordnungsgemäß nennen, einen Link zur Creative Commons Lizenz beifügen und angeben, ob Änderungen vorgenommen wurden.

Die in diesem Kapitel enthaltenen Bilder und sonstiges Drittmaterial unterliegen ebenfalls der genannten Creative Commons Lizenz, sofern sich aus der Abbildungslegende nichts anderes ergibt. Sofern das betreffende Material nicht unter der genannten Creative Commons Lizenz steht und die betreffende Handlung nicht nach gesetzlichen Vorschriften erlaubt ist, ist für die oben aufgeführten Weiterverwendungen des Materials die Einwilligung des jeweiligen Rechteinhabers einzuholen.

(c) (1) 